\title{
Common Fixed Point Theorems in Cone Metric Spaces under General Contractive Conditions
}

\author{
O. P. Chauhan, N. Singh, D. Singh, L. N. Mishra
}

\begin{abstract}
In this article, some common fixed point theorems for two pairs of compatible mappings together with sub-sequential continuity (alternately sub-compatible mappings together with reciprocal continuity) are proved in the setting of cone metric spaces. Our results are new in this setting especially in view of the note given in Imdad et al. [15]. On the other hand some fixed point results for faintly compatible mappings are also established. Illustrative examples along with their pictorial representation are furnished to highlight the validity of the hypothesis of our results
\end{abstract}

Keywords: Cone metric spaces, sub-compatible maps, sub-sequential continuity, reciprocal continuity.

\section{Introduction}

Fixed point theory is one of the most useful and effective tools in several branches of mathematics which has enormous applications within as well as outside mathematics. Starting from the eminent Banach contraction principle [2], many authors have obtained its numerous generalizations and its applications in different directions ([5],[6],[21]-[24],[26]) .

In 2007, Haung and Zhang [13] introduced the notion of cone metric spaces as a generalization of metric spaces by replacing the real numbers by ordering Banach Space. Later on many authors generalized fixed point results of different contractive conditions in cone metric spaces, some of them appeared in ([7]-[12],[14],[16],[17],[19],[20],[25],[29]).

In 1986, Jungck [18] introduced the notion of compatible mappings to generalize the idea of weak commutativity due to Sessa [30].

Manuscript received June 13, 2017; accepted November 15, 2017.

Naval Singh is with Department of Mathematics, Govt. Science and Commerce College, Benazeer, Bhopal, India; Deepak Singh is with Department of Applied Sciences NITTTR, Bhopal, Under the Ministry of HRD, Govt. of India; L. N. Mishra (corresponding author) is with the Department of Mathematics, Lovely Professional University, Punjab, India; Om Prakash Chauhan is with Department of Applied Mathematics, Jabalpur Engineering College, Jabalpur, India. 
In 1998, Pant [27] defined a new continuity condition known as reciprocal continuity and obtained a common fixed point theorem by owning the concept of compatibility in a metric space.

In 2009, Bouhadjera and Godet-Thobie [4] further enlarged the class of compatible (reciprocally continuous) pairs by introducing the concept of sub-compatibility (sub-sequential continuity) of pairs of mappings, which is substantially weaker than compatibility (reciprocal continuity). Afterward, Imdad et al. [15] improved the results of Bouhadjera and GodetThobie and showed that these results can easily be recovered by replacing sub-compatibility with compatibility or sub-sequential continuity with reciprocal continuity. Recently, the notion of faintly compatible mappings announced by Bisht et al. [3] as an improvement of conditionally compatible mappings.

The aim of this paper is to prove some common fixed point theorems for two pairs of self-mappings by using the notions of compatibility and sub-sequential continuity (alternately sub-compatibility and reciprocal continuity) satisfying general contractive conditions in a cone metric space.

\section{Preliminaries}

For basic terms and notation in cone metric space we refer to [13].

In the following we always suppose $E$ to be a Banach space, $P$ is a cone in $E$ with int $P \neq \emptyset$ and $\precsim$ is a partial ordering with respect to $P$.

Definition 1. [13] Let $X$ be a non empty set. Suppose the mapping $d: X \times X \rightarrow E$ satisfies

(d1) $0 \prec d(x, y)$ for all $x, y \in X$ and $d(x, y)=0$ if and only if $x=y$;

(d2) $d(x, y)=d(y, x)$ for all $x, y \in X$

(d3) $d(x, y) \precsim d(x, z)+d(y, z)$ for all $x, y, z \in X$.

Then $d$ is called a cone metric on $X$, and $(X, d)$ is called a cone metric space.

For convergence, Cauchy sequence and completeness we refer to [13].

Lemma 1. [13] Let $(X, d)$ be a cone metric space, $\left\{x_{n}\right\}$ be a sequence in $X$. If $\left\{x_{n}\right\}$ converges to $x$, then $\left\{x_{n}\right\}$ is a Cauchy sequence.

Lemma 2. [13] Let $(X, d)$ be a cone metric space, $P$ be a normal cone with normal constant $K$. Let $\left\{x_{n}\right\}$ be a sequence in $X$ Then $\left\{x_{n}\right\}$ is a Cauchy sequence if and only if $d\left(x_{n}, x\right) \rightarrow$ $0(n, m \rightarrow \infty)$.

Definition 2. [1] Let $A$ and $S$ be self mappings of a set $X$. If $w=A x=S x$ for some $x$ in $X$ is called a coincidence point of $A$ and $S$, and $w$ is called a point of coincidence of $A$ and $S$. 
Definition 3. [18] Let $A, S: X \rightarrow X$ be two self-mappings on a cone metric space $(X, d)$. The mappings $A$ and $S$ are said to be compatible if

$$
\lim _{n \rightarrow \infty} d\left(A S x_{n}, S A x_{n}\right)=0
$$

for each sequence $\left\{x_{n}\right\}$ in $X$ such that $\lim _{n \rightarrow \infty} A x_{n}=\lim _{n \rightarrow \infty} S x_{n}=t$ for some $t \in X$

Definition 4. A pair $(A, S)$ of self-mappings on a cone metric space $(X, d)$ is said to be non-compatible if there exist a sequence $\left\{x_{n}\right\}$ in $X$ such that

$$
\lim _{n \rightarrow \infty} A x_{n}=\lim _{n \rightarrow \infty} S x_{n}=x, \text { for some } x \in X
$$

and $\lim _{n \rightarrow \infty} d\left(A S x_{n}, S A x_{n}\right) \neq 0$ or non-existent.

Definition 5. [28] A pair $(A, S)$ of self-mappings on a cone metric space $(X, d)$ is said to be conditionally compatible iff whenever the set of sequences $\left\{x_{n}\right\}$ satisfying $\lim _{n \rightarrow \infty} A x_{n}=$ $\lim _{n \rightarrow \infty} S x_{n}$, is non-empty, there exists a sequence $\left\{z_{n}\right\}$ in $X$ such that

$$
\lim _{n \rightarrow \infty} A z_{n}=\lim _{n \rightarrow \infty} S z_{n}=t, \text { for some } t \in X, \text { and } \lim _{n \rightarrow \infty} d\left(A S z_{n}, S A z_{n}\right)=0 .
$$

Definition 6. [3] A pair $(A, S)$ of self-mappings on a cone metric space $(X, d)$ is said to be faintly compatible if $(A, S)$ is conditionally compatible and $A$ and $S$ commute on a non empty subset of the set of coincidence points, whenever the set of coincidence points is nonempty.

Definition 7. [27]. A pair $(A, S)$ of self-mappings on a cone metric space $(X, d)$ is called reciprocally continuous iffor each sequence sequence $\left\{x_{n}\right\}$ in $X, \lim _{n \rightarrow \infty} A S x_{n}=A z$ and $\lim _{n \rightarrow \infty} S A x_{n}=$ $S z$, whenever $\lim _{n \rightarrow \infty} A x_{n}=\lim _{n \rightarrow \infty} S x_{n}=z$, for some $z \in X$.

One can easily verify that if two self-mappings are continuous, then they are clearly reciprocally continuous, but the converse statement does not hold good in general. Furthermore, in the setting of common fixed point theorems for compatible pairs of self-mappings satisfying contractive conditions, continuity of one of the mappings implies their reciprocal continuity but the converse is not true.

Definition 8. [4] A pair $(A, S)$ of self-mappings on a cone metric space $(X, d)$ is said to be sub-compatible if there exists a sequence $\left\{x_{n}\right\}$ such that

$$
\lim _{n \rightarrow \infty} A x_{n}=\lim _{n \rightarrow \infty} S x_{n}=z \text { and } \lim _{n \rightarrow \infty} d\left(A S x_{n}, S A x_{n}\right)=0 .
$$


Definition 9. [4] A pair $(A, S)$ of self-mappings on a metric space $(X, d)$ is called subsequentially continuous if there exists a sequence $\left\{x_{n}\right\}$ in $X$ such that $\lim _{n \rightarrow \infty} A x_{n}=\lim _{n \rightarrow \infty} S x_{n}=z$, for some $z \in X$ such that

$$
\lim _{n \rightarrow \infty} A S x_{n}=A z \text { and } \lim _{n \rightarrow \infty} S A x_{n}=S z .
$$

If two self-mappings $A$ and $S$ are both continuous, hence reciprocally continuous mappings but are not sub-sequentially continuous.

\section{Main Results}

Our main results runs as follows.

Theorem 1. Let $A, B, S$ and $T$ be self mappings on a cone metric space $(X, d)$, where $d$ : $X \times X \rightarrow E$. Suppose that the pairs $(A, S)$ and $(B, T)$ are compatible and sub-sequentially continuous (alternately sub compatible and reciprocal continuous), satisfying the inequality

$$
\begin{gathered}
d(A x, B y) \precsim k_{1}(d(S x, T y)+d(A x, S x))+k_{2}(d(S x, T y)+d(B y, T y)) \\
+k_{3}\left(d(S x, T y)+\frac{d(S x, B y)+d(A x, T y)}{2}\right)
\end{gathered}
$$

for all $x, y \in X$, where $k_{1}, k_{2}, k_{3} \geq 0$ and $k_{1}+k_{2}+2 k_{3}<1$.

Then $A, B, S$ and $T$ have a unique common fixed point in $X$.

Proof. If the pair of mapping $(A, S)$ (also $(B, T)$ ) is sub-sequentially continuous and compatible, there exists a sequence $\left\{x_{n}\right\}$ in $X$ such that

$$
\lim _{n \rightarrow \infty} A x_{n}=\lim _{n \rightarrow \infty} S x_{n}=t, \text { for some } t \in X,
$$

and

$$
\lim _{n \rightarrow \infty} d\left(A S x_{n}, S A x_{n}\right)=d(A t, S t)=0
$$

so that $A t=S t$.

Similarly, with respect to the pair $(B, T)$, there exists a sequence $\left\{y_{n}\right\}$ in $X$ such that

$$
\lim _{n \rightarrow \infty} B y_{n}=\lim _{n \rightarrow \infty} T y_{n}=z, \text { for some } z \in X,
$$

and

$$
\lim _{n \rightarrow \infty} d\left(B T y_{n}, T B y_{n}\right)=d(B z, T z)=0,
$$

so that $B z=T z$.

Hence $t$ is a coincidence point of the pair $(A, S)$, whereas $z$ is a coincidence point of the pair 
$(B, T)$.

Now we assert that $t=z$. If $t \neq z$ then using inequality (1) with $x=x_{n}$ and $y=y_{n}$, one gets

$$
\begin{aligned}
d\left(A x_{n}, B y_{n}\right) \precsim & k_{1}\left(d\left(S x_{n}, T y_{n}\right)+d\left(A x_{n}, S x_{n}\right)\right)+k_{2}\left(d\left(S x_{n}, T y_{n}\right)+d\left(B y_{n}, T y_{n}\right)\right) \\
& +k_{3}\left(d\left(S x_{n}, T y_{n}\right)+\frac{d\left(S x_{n}, B y_{n}\right)+d\left(A x_{n}, T y_{n}\right)}{2}\right) .
\end{aligned}
$$

Which on letting $n \rightarrow \infty$, reduces to

$$
\begin{aligned}
d(t, z) & \precsim k_{1}(d(t, z)+d(t, t))+k_{2}(d(t, z)+d(z, z))+ \\
& k_{3}\left(d(t, z)+\frac{d(t, z)+d(t, z)}{2}\right) \\
& \precsim k_{1} d(t, z)+k_{2} d(t, z)+2 k_{3} d(t, z) \\
& \precsim\left(k_{1}+k_{2}+2 k_{3}\right) d(t, z),
\end{aligned}
$$

which is a contradiction. Therefore $t=z$. Next to show that $A t=t$. On the contrary if $A t \neq t$, then from inequality (1) with $x=t$ and $y=y_{n}$, we have

$$
\begin{gathered}
d\left(A t, B y_{n}\right) \precsim k_{1}\left(d\left(S t, T y_{n}\right)+d(A t, S t)\right)+k_{2}\left(d\left(S t, T y_{n}\right)+d\left(B y_{n}, T y_{n}\right)\right)+ \\
k_{3}\left(d\left(S t, T y_{n}\right)+\frac{d\left(S t, B y_{n}\right)+d\left(A t, T y_{n}\right)}{2}\right) .
\end{gathered}
$$

Taking the limit as $n \rightarrow \infty$, we get

$$
\begin{gathered}
d(A t, z) \precsim k_{1}(d(A t, z)+d(A t, A t))+k_{2}(d(A t, z)+d(z, z))+ \\
k_{3}\left(d(A t, z)+\frac{d(A t, z)+d(A t, z)}{2}\right)
\end{gathered}
$$

that is

$$
\begin{gathered}
d(A t, t) \precsim k_{1}(d(A t, t)+d(A t, A t))+k_{2}(d(A t, t)+d(t, t))+ \\
k_{3}\left(d(A t, t)+\frac{d(A t, t)+d(A t, t)}{2}\right)
\end{gathered}
$$

on simplification, we get

$$
d(A t, t) \precsim\left(k_{1}+k_{2}+2 k_{3}\right) d(A t, t),
$$

this is a contradiction. thus $A t=t$. Therefore $A t=S t=t$.

Now we show that $B t=t$. If $B t \neq t$ then using inequality (1) with $x=x_{n}$ and $y=z$, we have

$$
\begin{gathered}
d\left(A x_{n}, B z\right) \precsim k_{1}\left(d\left(S x_{n}, T z\right)+d\left(A x_{n}, S x_{n}\right)\right)+k_{2}\left(d\left(S x_{n}, T z\right)+d(B z, T z)\right)+ \\
k_{3}\left(d\left(S x_{n}, T z\right)+\frac{d\left(S x_{n}, B z\right)+d\left(A x_{n}, T z\right)}{2}\right) .
\end{gathered}
$$


Making on $n \rightarrow \infty$, one gets

$$
\begin{aligned}
d(t, B t) & \precsim k_{1}(d(t, B t)+d(t, t))+k_{2}(d(t, B t)+d(B t, B t)) \\
& +k_{3}\left(d(t, B t)+\frac{d(t, B t)+d(t, B t)}{2}\right) \\
& \precsim\left(k_{1}+k_{2}+2 k_{3}\right) d(t, B t),
\end{aligned}
$$

a contradiction . Hence $B t=t$. Therefore $A t=S t=B t=T t=t$, that is, $t$ is a common fixed point of $A, B, S$ and $T$.

Uniqueness: For uniqueness suppose that $w$ is another fixed point of mappings $A, B, S$ and $T$ which is different from $t$. Thus $A w=S w=B w=T w=w$. From inequality (1) with $x=t$ and $y=w$, we have

$$
\begin{aligned}
d(A t, B w) & \precsim k_{1}(d(S t, T w)+d(A t, S t))+k_{2}(d(S t, T w)+d(B w, T w)) \\
& +k_{3}\left(d(S t, T w)+\frac{d(S t, B w)+d(A t, B w)}{2}\right) \\
d(t, w) & \precsim k_{1}(d(t, w)+d(t, t))+k_{2}(d(t, w)+d(w, w)) \\
& +k_{3}\left(d(t, w)+\frac{d(t, w)+d(t, w)}{2}\right) \\
& \precsim\left(k_{1}+k_{2}+2 k_{3}\right) d(t, w),
\end{aligned}
$$

which is a contradiction. Hence $t=w$. Thus the common fixed point is unique.

Now suppose that the mappings $(A, S)$ and $(B, T)$ are sub-compatible and reciprocal continuous. Then there exists a sequence $\left\{x_{n}\right\}$ in $X$ such that

$$
\lim _{n \rightarrow \infty} A x_{n}=\lim _{n \rightarrow \infty} S x_{n}=t, \text { for some } t \in X,
$$

and

$$
\lim _{n \rightarrow \infty} d\left(A S x_{n}, S A x_{n}\right)=d(A t, S t)=0,
$$

whereas in respect of the pair $(B, T)$, there exists a sequence $\left\{y_{n}\right\}$ in $X$ with

$$
\lim _{n \rightarrow \infty} B y_{n}=\lim _{n \rightarrow \infty} T y_{n}=z, \text { for some } z \in X,
$$

and

$$
\lim _{n \rightarrow \infty} d\left(B T y_{n}, T B y_{n}\right)=d(B z, T z)=0 .
$$

Therefore, $A t=S t$ and $B z=T z$, that is, $t$ is a coincidence point of the pair $(A, S)$ whereas $z$ is a coincidence point of the pair $(B, T)$. The rest of part the proof can be completed easily.

If we set $A=B$ and $S=T$ in Theorem 1, we obtain the corollary for two mappings. 
Corollary 1. Let $A$ and $S$ be self mappings on a cone metric space $(X, d)$, where $d: X \times X \rightarrow$ $E$. Suppose that the pair $(A, S)$ is compatible and sub-sequentially continuous (alternately sub compatible and reciprocal continuous), satisfying the inequality

$$
\begin{gathered}
d(A x, A y) \precsim k_{1}(d(S x, S y)+d(A x, S x))+k_{2}(d(S x, S y)+d(A y, S y))+ \\
k_{3}\left(d(S x, S y)+\frac{d(S x, A y)+d(A x, S y)}{2}\right)
\end{gathered}
$$

for all $x, y \in X$, where $k_{1}, k_{2}, k_{3} \geq 0$ and $k_{1}+k_{2}+2 k_{3}<1$.

Then $A$ and $S$ have a unique common fixed point in $X$.

Now we furnish two illustrative examples to highlight the utility of Theorem (1). First example is presented for compatible and sub-sequential continuous mappings

Example 1. Let $(X, d)$ be a cone metric space with partial ordering ${ }^{\prime} \leq{ }^{\prime}$ and $E=R^{2}, P=$ $\{(x, y) \in E \mid x, y>0\} \subset R^{2}, X=[0, \infty), d: X \times X \rightarrow E$, such that $d(x, y)=(|x-y|, \alpha|x-y|)$, where $\alpha \geq 0$ is a constant. Define the self mappings $A, B, S$ and $T$ by $A x=B x=\left\{\begin{array}{ll}\frac{x}{6}, & \text { if } x \in[0,1] \\ \frac{x+5}{6}, & \text { if } x \in(1, \infty)\end{array} \quad\right.$ and $\quad S x=T x= \begin{cases}\frac{x}{5}, & \text { if } x \in[0,1] \\ \frac{x+4}{5}, & \text { if } x \in(1, \infty) .\end{cases}$

Consider the sequence $\left\{x_{n}\right\}=\left\{\frac{1}{n}\right\}_{n \in N}$ in $X$. Then

$$
\lim _{n \rightarrow \infty} A x_{n}=\lim _{n \rightarrow \infty}\left(\frac{1}{6 n}\right)=0=\lim _{n \rightarrow \infty}\left(\frac{1}{5 n}\right)=\lim _{n \rightarrow \infty} S x_{n}
$$

and

$$
\begin{gathered}
\lim _{n \rightarrow \infty} A S x_{n}=\lim _{n \rightarrow \infty} A\left(\frac{1}{5 n}\right)=\lim _{n \rightarrow \infty}\left(\frac{1}{30 n}\right)=0=A(0) . \\
\lim _{n \rightarrow \infty} S A x_{n}=\lim _{n \rightarrow \infty} S\left(\frac{1}{6 n}\right)=\lim _{n \rightarrow \infty}\left(\frac{1}{30 n}\right)=0=S(0) . \\
\Rightarrow \lim _{n \rightarrow \infty} d\left(A S x_{n}, S A x_{n}\right)=0 .
\end{gathered}
$$

Consider another sequence $\left\{x_{n}\right\}=\left\{1+\frac{1}{n}\right\}_{n \in N}$ in $X$. Then

$$
\lim _{n \rightarrow \infty} A x_{n}=\lim _{n \rightarrow \infty}\left(1+\frac{1}{6 n}\right)=1=\lim _{n \rightarrow \infty}\left(1+\frac{1}{5 n}\right)=\lim _{n \rightarrow \infty} S x_{n}
$$

and

$$
\begin{aligned}
& \lim _{n \rightarrow \infty} A S x_{n}=\lim _{n \rightarrow \infty} A\left(1+\frac{1}{5 n}\right)=\lim _{n \rightarrow \infty}\left(1+\frac{1}{30 n}\right)=1 \neq A(1) . \\
& \lim _{n \rightarrow \infty} S A x_{n}=\lim _{n \rightarrow \infty} S\left(1+\frac{1}{6 n}\right)=\lim _{n \rightarrow \infty}\left(1+\frac{1}{30 n}\right)=1 \neq S(1) .
\end{aligned}
$$


Clearly, $\lim _{n \rightarrow \infty} d\left(A S x_{n}, S A x_{n}\right)=0$.

Thus, the pair $(A, S)$ is compatible as well as sub sequentially continuous but not reciprocally continuous (the same for the pair $(B, T)$ ).

Next we show that inequality (1) is satisfied.

$$
\begin{gathered}
d(A x, B y) \leq k_{1}(d(S x, T y)+d(A x, S x))+k_{2}(d(S x, T y)+d(B y, T y))+ \\
k_{3}\left(d(S x, T y)+\frac{d(S x, B y)+d(A x, T y)}{2}\right) .
\end{gathered}
$$

Following case are dealt in detail.

Case I: When $x, y \in[0,1]$,

$$
\begin{gathered}
d\left(\frac{x}{6}, \frac{y}{6}\right) \leq k_{1}\left(d\left(\frac{x}{5}, \frac{y}{5}\right)+d\left(\frac{x}{6}, \frac{x}{5}\right)\right)+k_{2}\left(d\left(\frac{x}{5}, \frac{y}{5}\right)+d\left(\frac{y}{6}, \frac{y}{5}\right)\right)+ \\
k_{3}\left(d\left(\frac{x}{5}, \frac{y}{5}\right)+\frac{d\left(\frac{x}{5}, \frac{y}{6}\right)+d\left(\frac{x}{6}, \frac{y}{5}\right)}{2}\right) . \\
\left(\frac{1}{6}|x-y|, \frac{1}{6} \alpha|x-y|\right) \leq k_{1}\left(\left(\frac{1}{5}|x-y|, \frac{1}{5} \alpha|x-y|\right)+\left(\frac{1}{30}|x|, \frac{1}{30} \alpha|x|\right)\right)+ \\
k_{2}\left(\left(\frac{1}{5}|x-y|, \frac{1}{5} \alpha|x-y|\right)+\left(\frac{1}{30}|y|, \frac{1}{30} \alpha|y|\right)\right)+ \\
k_{3}\left(\left(\frac{1}{5}|x-y|, \frac{1}{5} \alpha|x-y|\right)+\right. \\
\quad\left(\frac{1}{30}|6 x-5 y|, \frac{1}{30} \alpha|6 x-5 y|\right)+; 2\left(\frac{1}{30}|5 x-6 y|, \frac{1}{30} \alpha|5 x-6 y|\right) \\
\left(\frac{1}{6}, \frac{1}{6} \alpha\right)|x-y| \leq k_{1}\left(\left(\frac{1}{5}, \frac{1}{5} \alpha\right)|x-y|+\left(\frac{1}{30}, \frac{1}{30} \alpha\right)|x|\right)+ \\
k_{2}\left(\left(\frac{1}{5}, \frac{1}{5} \alpha\right)|x-y|+\left(\frac{1}{30}, \frac{1}{30} \alpha\right)|y|\right)+ \\
k_{3}\left(\left(\frac{1}{5}, \frac{1}{5} \alpha\right)|x-y|+\frac{\left(\frac{1}{30}, \frac{1}{30} \alpha\right)|6 x-5 y|+\left(\frac{1}{30}, \frac{1}{30} \alpha\right)|5 x-6 y|}{2}\right) .
\end{gathered}
$$

If $k_{1}=\frac{3}{5}, k_{2}=\frac{1}{50}, k_{3}=\frac{1}{5.3}$ and $\alpha \geq 0$. Clearly inequality (1) is satisfied for each $x, y \in[0,1]$.

Case II: When $x \in[0,1]$ and $y \in(1, \infty)$,

$$
\begin{aligned}
d\left(\frac{x}{6}, \frac{y+5}{6}\right) \leq & k_{1}\left(d\left(\frac{x}{5}, \frac{y+4}{5}\right)+d\left(\frac{x}{6}, \frac{x}{5}\right)\right)+ \\
& k_{2}\left(d\left(\frac{x}{5}, \frac{y+4}{5}\right)+d\left(\frac{y+5}{6}, \frac{y+4}{5}\right)\right)+ \\
& k_{3}\left(d\left(\frac{x}{5}, \frac{y+4}{5}\right)+\frac{d\left(\frac{x}{5}, \frac{y+5}{6}\right)+d\left(\frac{x}{6}, \frac{y+4}{5}\right)}{2}\right) .
\end{aligned}
$$


Calculating the same as in Case I, we conclude that Inequality (1) is satisfied for $x \in[0,1]$ and $y \in(1, \infty)$ and $k_{1}=\frac{3}{5}, k_{2}=\frac{1}{50}, k_{3}=\frac{1}{5.3}$ and $\alpha \geq 0$.

Case III: When $x \in(1, \infty)$ and $y \in[0,1]$,

$$
\begin{aligned}
d\left(\frac{x+5}{6}, \frac{y}{6}\right) \leq & k_{1}\left(d\left(\frac{x+4}{5}, \frac{y}{5}\right)+d\left(\frac{x+5}{6}, \frac{x+4}{5}\right)\right)+ \\
& k_{2}\left(d\left(\frac{x+4}{5}, \frac{y}{5}\right)+d\left(\frac{y}{6}, \frac{y}{5}\right)\right)+ \\
& k_{3}\left(d\left(\frac{x+4}{5}, \frac{y}{5}\right)+\frac{d\left(\frac{x+4}{5}, \frac{y}{6}\right)+d\left(\frac{x+5}{6}, \frac{y}{5}\right)}{2}\right) .
\end{aligned}
$$

For $k_{1}=\frac{3}{5}, k_{2}=\frac{1}{50}, k_{3}=\frac{1}{5.3}$ and $\alpha \geq 0$. Inequality 1 is satisfied for $x \in(1, \infty)$ and $y \in[0,1]$. Case IV: When $x, y \in(1, \infty)$,

$$
\begin{aligned}
d\left(\frac{x+5}{6}, \frac{y+5}{6}\right) \leq & k_{1}\left(d\left(\frac{x+4}{5}, \frac{y+4}{5}\right)+d\left(\frac{x+5}{6}, \frac{x+4}{5}\right)\right)+ \\
& k_{2}\left(d\left(\frac{x+4}{5}, \frac{y+4}{5}\right)+d\left(\frac{y+5}{6}, \frac{y+4}{5}\right)\right)+ \\
& k_{3}\left(d\left(\frac{x+4}{5}, \frac{y+4}{5}\right)+\frac{d\left(\frac{x+4}{5}, \frac{y+5}{6}\right)+d\left(\frac{x+5}{6}, \frac{y+4}{5}\right)}{2}\right) .
\end{aligned}
$$

If $k_{1}=\frac{3}{5}, k_{2}=\frac{1}{50}, k_{3}=\frac{1}{5.3}$ and $\alpha \geq 0$. Inequality 1 is satisfied for each $x, y \in(0, \infty)$.

Thus all the conditions of Theorem 1 are satisfied and 0 remains the unique common fixed point of the mappings $A, B, S$ and $T$, which is demonstrated by the following figure.

Next example validates the alternative hypothesis in Theorem 1.

Example 2. Let $(X, d)$ is a cone metric space with partial ordering ' $\leq{ }^{\prime}$ and $E=R^{2}, P=$ $\{(x, y) \in E \mid x, y>0\} \subset R^{2}, X=R, d: R \times R \rightarrow E$, where $R=(-\infty, \infty)$ such that $d(x, y)=$ $(|x-y|, \alpha|x-y|)$, where $\alpha \geq 0$ is a constant. Define the self mappings $A, B, S$ and $T$ by $A x=B x=\left\{\begin{array}{l}\frac{x}{3}, \quad \text { if } x \in(-\infty, 1) \\ 4 x-3, \text { if } x \in[1, \infty)\end{array}\right.$ and $S x=T x=\left\{\begin{array}{l}x+2, \text { if } x \in(-\infty, 1) \\ 3 x-2, \text { if } x \in[1, \infty) .\end{array}\right.$ Consider the sequence $\left\{x_{n}\right\}=\left\{1+\frac{1}{n}\right\}_{n \in N}$ in $X$. Then

$$
\lim _{n \rightarrow \infty} A x_{n}=\lim _{n \rightarrow \infty}\left(1+\frac{4}{n}\right)=1=\lim _{n \rightarrow \infty}\left(1+\frac{3}{n}\right)=\lim _{n \rightarrow \infty} S x_{n} .
$$

Also,

$$
\begin{aligned}
& \lim _{n \rightarrow \infty} A S x_{n}=\lim _{n \rightarrow \infty} A\left(1+\frac{3}{n}\right)=\lim _{n \rightarrow \infty}\left(1+\frac{12}{n}\right)=1=A(1), \\
& \lim _{n \rightarrow \infty} S A x_{n}=\lim _{n \rightarrow \infty} S\left(1+\frac{4}{n}\right)=\lim _{n \rightarrow \infty}\left(1+\frac{12}{n}\right)=1=S(1),
\end{aligned}
$$




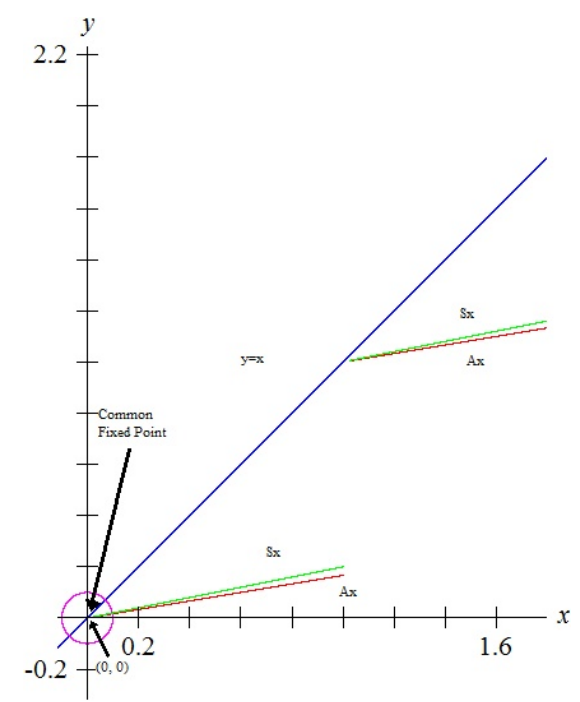

Fig. 1.

$$
\Rightarrow \lim _{n \rightarrow \infty} d\left(A S x_{n}, S A x_{n}\right)=0 .
$$

Consider another sequence $\left\{x_{n}\right\}=\left\{\frac{1}{n}-3\right\}_{n \in N}$ in $X$. Then

$$
\lim _{n \rightarrow \infty} A x_{n}=\lim _{n \rightarrow \infty}\left(\frac{1}{3 n}-1\right)=-1=\lim _{n \rightarrow \infty}\left(\frac{1}{n}-1\right)=\lim _{n \rightarrow \infty} S x_{n} .
$$

Next,

$$
\begin{gathered}
\lim _{n \rightarrow \infty} A S x_{n}=\lim _{n \rightarrow \infty} A\left(-1+\frac{1}{n}\right)=\lim _{n \rightarrow \infty}\left(\frac{-1}{3}+\frac{1}{3 n}\right)=\frac{-1}{3}=A(-1), \\
\lim _{n \rightarrow \infty} S A x_{n}=\lim _{n \rightarrow \infty} S\left(-1+\frac{1}{3 n}\right)=\lim _{n \rightarrow \infty}\left(1+\frac{1}{3 n}\right)=1=S(-1) .
\end{gathered}
$$

But, $\quad \lim _{n \rightarrow \infty} d\left(A S x_{n}, S A x_{n}\right) \neq 0$.

Thus, the pair $(A, S)$ is reciprocally continuous as well as sub compatible but not compatible (the same for the pair $(B, T)$ ). As in Example 1 it is easy to check that inequality (1) of Theorem1 satisfied with $k_{1}+k_{2}+2 k_{3}<1$ and $\alpha \geq 0$. Therefore all the conditions are satisfied. Here, 1 is a coincidence point as well as unique common fixed point of the mappings $A, B, S$ and $T$, which is given by the following figure.

Some more results are demonstrated with the different mapping conditions.

Theorem 2. Let $A, B, S$ and $T$ be self mappings on a cone metric space $(X, d)$, where $d$ : $X \times X \rightarrow E$.Suppose that the pairs $(A, S)$ and $(B, T)$ are non-compatible, faintly compatible, reciprocal continuous and satisfying the inequality (1).

Then $A, B, S$ and $T$ have a unique common fixed point in $X$. 


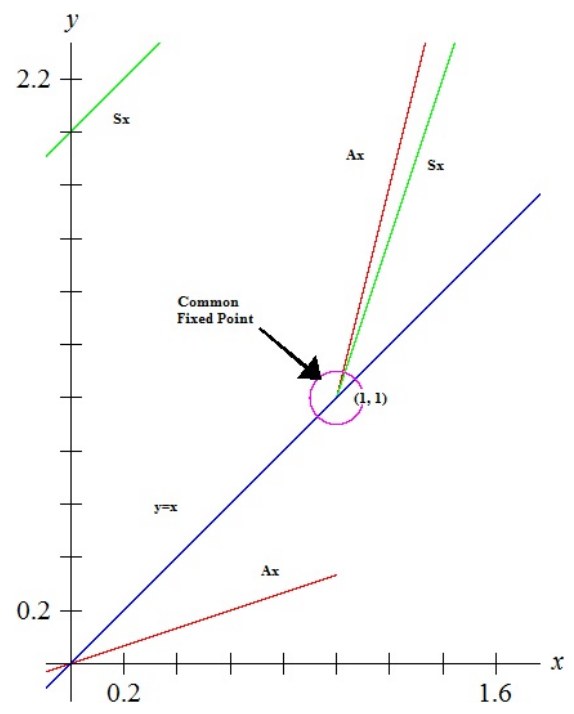

Fig. 2.

Proof. Since the pairs $(A, S)$ and $(B, T)$ are non-compatible, there exist sequences $\left\{x_{n}\right\}$ and $\left\{y_{n}\right\}$ in $X$, such that

$$
\lim _{n \rightarrow \infty} A x_{n}=\lim _{n \rightarrow \infty} S x_{n}=t_{1}, \text { for some } t_{1} \in X
$$

and $d\left(A S x_{n}, S A x_{n}\right) \neq 0$ or non existent. Also

$$
\lim _{n \rightarrow \infty} B y_{n}=\lim _{n \rightarrow \infty} T y_{n}=t_{2}, \text { for some } t_{2} \in X
$$

and $d\left(B T y_{n}, T B y_{n}\right) \neq 0$ or non existent.

Since the pairs $(A, S)$ and $(B, T)$ are faintly compatible, then conditionally compatibility of $(A, S)$ and $(B, T)$ implies that there exist sequences $\left\{z_{n}\right\}$ and $\left\{z_{n^{\prime}}\right\}$ in $X$ satisfying

$$
\lim _{n \rightarrow \infty} A z_{n}=\lim _{n \rightarrow \infty} S z_{n}=u, \text { for some } u \in X,
$$

such that $d\left(A S z_{n}, S A z_{n}\right)=0$. Also

$$
\lim _{n \rightarrow \infty} B z_{n^{\prime}}=\lim _{n \rightarrow \infty} T z_{n^{\prime}}=v, \text { for some } v \in X,
$$

such that $d\left(B T z_{n^{\prime}}, T B z_{n^{\prime}}\right)=0$.

As the pairs $(A, S)$ and $(B, T)$ are reciprocally continuous, we get

$$
\lim _{n \rightarrow \infty} A S z_{n}=A u, \lim _{n \rightarrow \infty} S A z_{n}=S u \text { and } s o A u=S u .
$$


Also,

$$
\lim _{n \rightarrow \infty} B T z_{n^{\prime}}=B v, \lim _{n \rightarrow \infty} T B z_{n^{\prime}}=T v \text { and so } B v=T v .
$$

Since pairs $(A, S)$ and $(B, T)$ are faintly compatible, we get

$$
A S u=S A u \text { and } s o A A u=A s u=S A u=S S u,
$$

and also,

$$
B T v=T B v \text { and } s o B B v=B T v=T B v=T T v .
$$

Now we assert that $A u=B v$. If not then using inequality (1) with $x=u$ and $y=v$

$$
\begin{aligned}
d(A u, B v) \precsim & k_{1}(d(S u, T v)+d(A u, S u))+k_{2}(d(S u, T v)+d(B v, T v)) \\
& +k_{3}\left(d(S u, T v)+\frac{d(S u, B v)+d(A u, T v)}{2}\right) \\
\precsim & k_{1}(d(A u, B v)+d(A u, A u))+k_{2}(d(A u, B v)+d(B v, B v)) \\
& +k_{3}\left(d(A u, B v)+\frac{d(A u, B v)+d(A u, B v)}{2}\right)
\end{aligned}
$$

$d(A u, B v) \precsim\left(k_{1}+k_{2}+2 k_{3}\right) d(A u, B v)$, is a contradiction. (since $k_{1}+k_{2}+2 k_{3}<1$.) Therefore, $A u=B v$.

Now we claim that $A A u=A u$. On contrary suppose $A A u \neq A u$ then using inequality (1) with $x=A u$ and $y=v$

$$
\begin{aligned}
d(A A u, B v) \precsim & k_{1}(d(S A u, T v)+d(A A u, S A u))+k_{2}(d(S A u, T v)+d(B v, T v)) \\
& +k_{3}\left(d(S A u, T v)+\frac{d(S A u, B v)+d(A A u, T v)}{2}\right) \\
\precsim & k_{1}(d(A A u, B v)+d(A A u, A A u))+k_{2}(d(A A u, B v)+d(B v, B v)) \\
& +k_{3}\left(d(A A u, B v)+\frac{d(A A u, B v)+d(A A u, B v)}{2}\right)
\end{aligned}
$$

$d(A u, B v) \precsim\left(k_{1}+k_{2}+2 k_{3}\right) d(A A u, B v)$, is a contradiction.

Hence, $A A u=B v$. Therefore $A A u=B v=A u$.

Next to show that $A u=B B v$, utilizing inequality (1) with $x=u$ and $y=B v$

$$
\begin{aligned}
d(A u, B B v) \precsim & k_{1}(d(S u, T B v)+d(A u, S u))+k_{2}(d(S u, T B v)+d(B B v, T B v)) \\
& +k_{3}\left(d(S u, T B v)+\frac{d(S u, B B v)+d(A u, T B v)}{2}\right) \\
\precsim & k_{1}(d(A u, B B v)+d(A u, A u))+k_{2}(d(A u, B B v)+d(B B v, B B 3 v)) \\
& +k_{3}\left(d(A u, B B v)+\frac{d(A u, B B v)+d(A u, B B v)}{2}\right.
\end{aligned}
$$


$d(A u, B B v) \precsim\left(k_{1}+k_{2}+2 k_{3}\right) d(A u, B B v)$, this is a contradiction.

Hence $A u=B B v$. Therefore $B B v=A u=B v$.

Now we have $A A u=S A u=A u, A u=B B v=B A u$ and $A u=T B v=T A u$ since $B v=A u$.

Hence $A A u=S A u=B A u=T A u=A u$, that is $A u$ is a common fixed point of $A, B, S$ and $T$.

Uniqueness of the fixed point is an easy consequence of (1).

This completes the Proof.

If we set $A=B$ and $S=T$ in Theorem 2, we obtain the corollary for two mappings.

Corollary 2. Let $A$ and $S$ be self mappings on a cone metric space $(X, d)$, where $d: X \times$ $X \rightarrow E$. Suppose that the pair $(A, S)$ is non-compatible, faintly compatible and reciprocal continuous also satisfying the inequality

$$
\begin{gathered}
d(A x, A y) \precsim k_{1}[d(S x, S y)+d(A x, S x)]+k_{2}[d(S x, S y)+d(A y, S y)] \\
+k_{3}\left[d(S x, S y)+\frac{d(S x, A y)+d(A x, S y)}{2}\right] .
\end{gathered}
$$

for all $x, y \in X$, where $k_{1}, k_{2}, k_{3} \geq 0$ and $k_{1}+k_{2}+2 k_{3}<1$.

Then $A$ and $S$ have a unique common fixed point in $X$.

Now, the following example is furnished to highlight the utility of Theorem 2 when involved pair of mappings are non-compatible,Faintly compatible and reciprocal continuous.

Example 3. Let $(X, d)$ is a cone metric space with partial ordering ' ${ }^{\prime}{ }^{\prime}$ and $E=R^{2}, P=$ $\{(x, y) \in E \mid x, y>0\} \subset R^{2}, X=[0,4], d: X \times X \rightarrow E$, such that

$$
d(x, y)=(|x-y|, \alpha|x-y|), \text { where } \alpha \geq 0 \text { is a constant } .
$$

Define the self mappings $A, B, S$ and $T$ by

$$
A x=B x=\left\{\begin{array}{ll}
2, & \text { if } x \leq 2 \\
4, & \text { if } x>2
\end{array} \text { and } S x=T x= \begin{cases}4-x, & \text { if } x \leq 2 \\
8, & \text { if } x>2 .\end{cases}\right.
$$

Consider the sequence $\left\{x_{n}\right\}=\left\{2-\frac{1}{n}\right\}_{n \in N}$ in $X$. Then

$$
\lim _{n \rightarrow \infty} A x_{n}=\lim _{n \rightarrow \infty}(2)=2=\lim _{n \rightarrow \infty}\left(2+\frac{1}{n}\right)=\lim _{n \rightarrow \infty} S x_{n}
$$

and

$$
\begin{gathered}
\lim _{n \rightarrow \infty} A S x_{n}=\lim _{n \rightarrow \infty} A\left(2+\frac{1}{n}\right)=4, \\
\lim _{n \rightarrow \infty} S A x_{n}=\lim _{n \rightarrow \infty} S(2)=2, \\
\Rightarrow \quad \lim _{n \rightarrow \infty} d\left(A S x_{n}, S A x_{n}\right) \neq 0 .
\end{gathered}
$$


Therefore $(A, S)$ is non-compatible. Consider another sequence $\left\{y_{n}\right\}=\{2\}_{n \in N}$ in $X$. Then

$$
\lim _{n \rightarrow \infty} A y_{n}=2=\lim _{n \rightarrow \infty} S y_{n}
$$

and

$$
\begin{aligned}
& \lim _{n \rightarrow \infty} A S y_{n}=\lim _{n \rightarrow \infty} A(2)=2, \\
& \lim _{n \rightarrow \infty} S A y_{n}=\lim _{n \rightarrow \infty} S(2)=2 .
\end{aligned}
$$

Clearly, $\lim _{n \rightarrow \infty} d\left(A S x_{n}, S A x_{n}\right)=0$ and the pair $(A, S)$ commute at their coincidence point $2 \in$ $X$. Thus, the pair $(A, S)$ is faintly compatible.

Let $\left\{x_{n}\right\} \in X$ be such that $\lim _{n \rightarrow \infty} A x_{n}=z=\lim _{n \rightarrow \infty} S x_{n}$ in $X$.

Then $\left\{x_{n}\right\}=2=z$ and $\lim _{n \rightarrow \infty} A S x_{n}=A z, \lim _{n \rightarrow \infty} S A x_{n}=S z$.

Therefore $(A, S)$ is reciprocally continuous.(Consequently the pair of mappings $(B, T)$ is also non-compatible, faintly compatible and reciprocally continuous).

Next we show that inequality (1) is satisfied.

Before discussing different cases, one needs to notice that

$$
0 \leq d(A x, B y), d(S x, T y), d(A x, S x), d(B y, T y), d(S x, B y), d(A x, T y), \forall x, y \in X .
$$

It is sufficient to show that $d(A x, A y) \leq k_{1} d(S x, S y)$ with $k_{1}=\frac{3}{5}$ and $k_{2}, k_{3} \geq 0$ such that $k_{1}+k_{2}+2 k_{3}<1$.

Following cases for $x, y \in X$ are dealt in detail.

Case I. For $x, y \leq 2$,

$$
\begin{aligned}
d(A x, A y)=d(2,2)=(0, \alpha .0) & \leq \frac{3}{5} d(S x, S y)=\frac{3}{5} d(4-x, 4-y) \\
& =\frac{3}{5}(|x-y|, \alpha|x-y|)
\end{aligned}
$$

Case II. For $x, y>2$,

$$
d(A x, A y)=d(4,4)=(0, \alpha .0) \leq \frac{3}{5} d(S x, S y)=\frac{3}{5} d(8,8)=(0, \alpha .0) .
$$

Case III. For $x \leq 2, y>2$,

$$
\begin{aligned}
d(A x, A y)=d(2,4)=(2, \alpha .2) & \leq \frac{3}{5} d(S x, S y)=\frac{3}{5} d(4-x, 8) \\
& =\frac{3}{5}(|x+4|, \alpha|x+4|) .
\end{aligned}
$$


Case IV. For $x>2, y \leq 2$,

$$
\begin{aligned}
d(A x, A y)=d(4,2)=(2, \alpha .2) & \leq \frac{3}{5} d(S x, S y)=\frac{3}{5} d(8,4-y) \\
& =\frac{3}{5}(|y+4|, \alpha|y+4|) .
\end{aligned}
$$

Thus all the conditions are satisfied. Therefore, the mappings $A, B, S$ and $T$ have a unique common fixed point $x=2$, which is shown by the following figure.

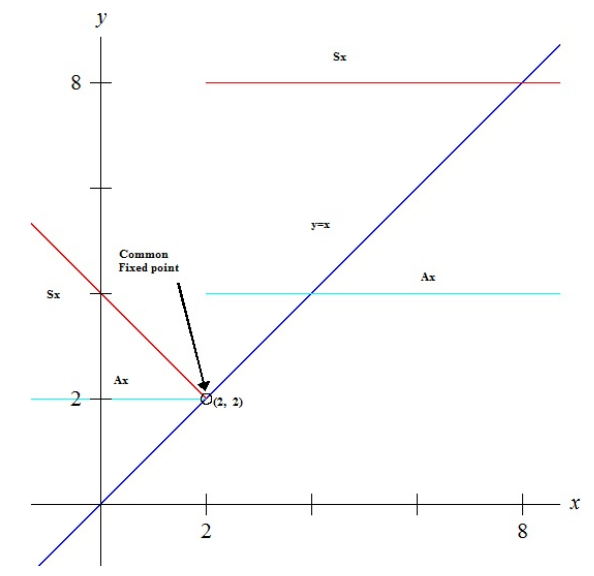

Fig. 3.

\section{Acknowledgments}

The authors would like to thank the anonymous referee for his/her comments that helped us to improve this article.

\section{References}

[1] M. Abbas, G. Jungck, Common fixed point results for non commuting mappings without continuity in cone metric spaces, Journal of Mathematical Analysis and Applications, 341 (1), (2008) pp. 416-420.

[2] S. Banach, Sur les operations dans les ensembles abstraits et leur application aux equations integrales, Fundamenta Mathematicae, Vol. 3, No. 1, (1922) pp. 133-181.

[3] R. K. Bisht, N. Shahzad, Faintly compatible mappings and common fixed points, Fixed Point Theory and Applications, 2013:156, 2013.

[4] H. Bouhadjera, C. Godet-Thobie, Common fixed point theorems for pairs of subcompatible maps, old version, 2009, http://arxiv.org/abs/0906.3159. 
[5] Lj. B. Ćirić, On a family of contractive maps and fixed points, Publications del Institut Mathematique, Vol. 17, No. 31, (1974) pp. 45-51.

[6] Lj. B. Ćirić, A generalization of Banachs contraction principle, Proceedings of the American Mathematical Society, Vol. 45, (1974) pp. 267-273.k

[7] S. H. Cho, J. S. Bae, Common fixed point theorem for mappings satisfying property (E.A.) on cone metric spaces, Mathematical and Computer Modelling, 53, (2011) pp. 945-951.

[8] Y. J. Cho, R. Sadati, S. Wang, Common fixed point theorems on generalized distance in ordered cone metric spaces, Comput. Math. Appl. 61, (2011) pp. 1254-1260.

[9] Deepmala, A Study on Fixed Point Theorems for Nonlinear Contractions and its Applications, Ph.D. Thesis (2014), Pt. Ravishankar Shukla University, Raipur (Chhatisgarh) India 492010.

[10] Z. M. Fadail, Abd G. B. Ahmad, S. Radenović, and M. Rajovic, On mixed g-monotone and w-compatible mappings in ordered cone b-metric spaces, Math Sci (2015) 9:161172.

[11] Z. M. Fadail, G. S. Rad, V. Ozturk and S. Radenović, Some on coupled, tripled and n-tupled fixed points theorems in ordered abstract metric spaces, Far East Journal of Mathematical Sciences, 97, 7, 2015, pp. 809-839.

[12] Y. Han, S. Xu, Some new theorems of expanding mappings without continuity in cone metric spaces, Fixed point Theory and Appl., 2013:3, 2013.

[13] L. G. Huang, X. Zhang, Cone metric spaces and fixed point theorems of contractive mappings, Journal of Mathematical Analysis and Applications, Vol. 332 (2), (2007) pp 468-476.

[14] H. Huang, Z. Kadelburg, S. Radenović, A note on some recent results about multivalued mappings in TVS-cone metric spaces, Accepted in J. Adv. Math. Stud. /25/11/2015/.

[15] M. Imdad, J. Ali, M. Tanveer, Remarks on some recent metrical common fixed point theorems, Applied Mathematics Letters, Vol. 24, No. 7, (2011) pp. 1165-1169.

[16] S. Janković, Z. Kadelburg, S. Radenović, On cone metric spaces: a survay, Nonlinear Analysis, 74 (2011) pp. 2591-2601.

[17] S. Janković, Z. Golubović, S. Radenović, Compatible and weakly compatible mappings in cone metric spaces, Mathematical and Computer Modeling 52 (2010) pp. 1728-1738.

[18] G. Jungck, Compatible mappings and common fixed points, International Journal of Mathematics and Mathematical Sciences, Vol. 9, No. 4, (1986) pp. 771-779.

[19] Z. Kadelburg, S. Radenović, V. Rakocević, A note on the equivalence of some metric and cone metric fixed point results, Appl. Math. Lett. 24, (2011), pp. 370-374.

[20] Z. Kadelburg, S. Radenović, A note on various types of cones and fixed point results in cone metric spaces, Asian Journal of Mathematics and Applications, Vol. 2013, Article ID ama0104, 7 pages.

[21] L. N. Mishra, S. K. Tiwari, V. N Mishra, I.A. Khan, Unique Fixed Point Theorems for Generalized Contractive Mappings in Partial Metric Spaces, Journal of Function Spaces, Vol. 2015 (2015),Article ID 960827.

[22] L. N. Mishra, S. K. Tiwari, V. N Mishra, Fixed point theorems for generalized weakly Scontractive mappings in partial metric spaces, Journal of Applied Analysis and Computation, Vol. 5, No. 4, (2015) pp. 600-612. 
Common Fixed Point Theorems in Cone Metric Spaces under General Contractive Conditions 149

[23] V. N. Mishra, Some Problems on Approximations of Functions in Banach Spaces, Ph.D. Thesis, (2007), Indian Institute of Technology, Roorkee - 247 667, Uttarakhand, India.

[24] V. N. Mishra, M. L. Mittal, U. Singh, On best approximation in locally convex space, Varhmihir Journal of Mathematical Sciences India, Vol. 6, No.1, (2006) pp. 43-48.

[25] F. Moradlou, P. Salimi, S. Radenović, Implicit Relation and Eldestein-Suzuki Type Fixed Point Results in Cone Metric Spaces, Applied Mathematics E-Notes, 14 (2014), pp. 1-12.

[26] H. K. Pathak and Deepmala, Common fixed point theorems for PD-operator pairs under Relaxed conditions with applications, Journal of Computational and App. Mathematics, 239 (2013), pp. 103-113.

[27] R. P. Pant, Common fixed points of four mappings, Bull. Calcutta Math. Soc., 90 (1998), 281286.

[28] R. P. Pant and R. K. Bisht, Occasionally weakly compatible mappings and fixed points, Bull. Belg. Math. Soc. Simon Stevin, 19 (2012), pp. 655-661.

[29] V.Ć. Rajić, S. Radenović, A note on tripled fixed point of w-compatible mappings in tvs-cone metric spaces, Thai Journal of Mathematics, 12 (2014) 3:717-728.

[30] S. Sessa, On a weak commutativity condition of mappings in fixed point considerations, Publications de l'Institut Mathematique, Vol. 32, No. 46, (1982), pp. 149-153. 